\title{
Cutaneous Malignant Melanoma Metastasis to the Pseudophakic Lens Capsule with Associated Granulomatous Intraocular Inflammation
}

\author{
Hibba Quhilla Hardeep S. Mudhar ${ }^{b} \quad$ Kurt Spiteri Cornish ${ }^{a} \quad$ lan G. Rennie ${ }^{a}$ \\ ${ }^{a}$ Department of Ophthalmology, Royal Hallamshire Hospital, Sheffield, UK; ${ }^{b}$ National Specialist Ophthalmic \\ Pathology Service (NSOPS), Department of Histopathology, Royal Hallamshire Hospital, Sheffield, UK
}

\section{Established Facts}

- Neoplastic pathology rarely masquerades as non-infectious uveitis and a high index of suspicion should be maintained in atypical cases that fail to respond to standard therapy.

- Full diagnostic pars plana vitrectomy is superior to core vitreous biopsy and vitreous tap for histopathological diagnosis.

\section{Novel Insights}

- This is the first case confirming cutaneous melanoma metastasis to the posterior lens capsule, in a seeing eye, based on diagnostic vitrectomy and capsulotomy.

- Granulomatous intraocular inflammation can occur concurrently with intraocular cutaneous melanoma metastasis.

\section{Keywords}

Malignant melanoma · Posterior capsule · Uveitis · Granulomatous inflammation · Metastasis · Cutaneous melanoma $\cdot$ Diagnostic vitrectomy

\section{Abstract \\ Intraocular cutaneous melanoma metastasis (ICMM) is a rare event, accounting for only $5 \%$ of all metastases to the eye and orbit. The vast majority of such metastases primarily af- fect the choroid and vitreoretinal structures. Only three pre-}

vious cases of predominant lens structure ICMM have been reported in the literature. Histological examination, in all three past cases, was performed on enucleation specimens of painful blind eyes. We present the first case of ICMM to the lens capsule in a comfortable, seeing, pseudophakic eye. This was histologically confirmed following diagnostic pars plana vitrectomy and capsulotomy, and was found to be associated with background granulomatous intraocular inflammation. The potential causes of the granulomatous inflammation are discussed.

(c) 2020 S. Karger AG, Base karger@karger.com

(C) 2020 S. Karger AG, Basel

www.karger.com/oop

Karger"
Hibba Quhill

Department of Ophthalmology

Royal Hallamshire Hospital

Glossop Road, Sheffield S10 2JF (UK)

Hibba.quhill@nhs.net 


\section{Introduction}

Intraocular cutaneous melanoma metastasis (ICMM) is a rare event, accounting for only $5 \%$ of all metastases to the eye and orbit [1]. The majority of metastases affect the uvea (59\%) and vitreous (28\%) [1], but it is exceedingly rare to have predominant involvement of the lens structures in the absence of a discrete intraocular mass. Only three previous cases have been reported in the literature, and the histology described was performed on enucleation specimens of painful blind eyes. Two described lens capsule involvement in phakic eyes with diffuse ICMM $[2,3]$, and the third reported ICMM adherent to the pseudophakic lens surface and posterior capsule [4]. We present the first case of ICMM to the lens capsule in a comfortable, seeing eye. This was histologically confirmed by diagnostic pars plana vitrectomy (PPV) and capsuloto$\mathrm{my}$, and was associated with background granulomatous intraocular inflammation.

\section{Case Presentation}

An 80-year-old-man, who was being followed up routinely for a regressed peripapillary choroidal neovascular membrane, was found incidentally to have anterior uveitis reducing his vision from his baseline of $6 / 6$ to $6 / 9$ in the right eye. His past ocular history included left amblyopia (best recorded vision of 6/24), bilateral pseudophakia, and six intravitreal injections of ranibizumab to treat the choroidal neovascular membrane in the right eye, 1 year previously. His significant past medical history included BRAFV600E mutated cutaneous malignant melanoma of the left groin excised 3 years prior to his ophthalmic presentation. Histology of the cutaneous lesion showed complete excision of a superficial spreading melanoma, with an invasive vertical growth phase, Breslow thickness of $1.5 \mathrm{~mm}$, without ulceration. This corresponded to pathological stage pT2a. He was known to have metastatic disease involving regional lymph nodes, bladder wall, and the right endobronchial area at the time of his referral to the ophthalmic services. He had not received any systemic oncological treatment, but was under close surveillance with interval computerized tomography of the chest, abdomen, and pelvis.

Over the following months, despite standard treatment of his right anterior uveitis with topical steroids and mydriatics, the intraocular inflammation deteriorated. The patient developed 'mutton fat' granulomatous keratic precipitates and significant vitreous haze, dropping the vision further to $6 / 24$. The differential of a masquerade syndrome was considered due to the atypical clinical course, and the patient underwent a vitreous 'tap' (core vitreous sample using a needle and syringe through the pars plana) 4 months after the initial detection of intraocular cells. The vitreous biopsy was paucicellular and only showed non-specific inflammation.

One month later, the patient presented with spontaneous hyphaema of the right eye and associated raised intraocular pressure. The vision had fallen to hand movements. Following standard management and partial resolution of the hyphaema, examination revealed mild rubeosis at the pupillary margin and pigmentation of the iridocorneal angle on gonioscopy (Fig. 1d). The suspicion of an intraocular melanomatous process was again raised, and the patient was referred to a tertiary centre.

Ocular oncological examination confirmed the presence of right mild iris rubeosis and detected brown pigmentation on the anterior and posterior lens capsule (Fig. 1a, c, and e). The left eye was normal (Fig. 1b). The vitreous was cellular containing nonpigmented cells, but no discrete intraocular mass was seen (Fig. 1f). There was renewed suspicion of ICMM to the lens capsule and possibly vitreous, so the patient underwent diagnostic PPV and surgical posterior capsulotomy using the vitrectomy cutter. The whole cassette was sent for histopathological examination, as recommended by Patel et al. [5].

The sample underwent cell block preparation and revealed pigmented and non-pigmented cytologically atypical cells alongside and adherent to pieces of lens capsule (Fig. 2a, b). There were associated inflammatory cells comprising lymphocytes and macrophages. Immunohistochemistry showed that some of the pigmented and non-pigmented cells were positive for Melan A (Fig. 2c) and Sox10 (Fig. 2d), which are melanocytic differentiation markers, confirming the diagnosis of metastatic cutaneous melanoma. The Sox10 showed groups of unstained cells that were suspicious for granulomas (Fig. 2d). The presence of the latter was confirmed by CD68 immunohistochemistry (macrophage marker) that revealed macrophages had congregated to form definite granulomas (Fig. 2e). The overall interpretation was of ICMM on a granulomatous inflammatory background.

The PPV proved both diagnostic and therapeutic as the visual axis was cleared, at least for the short to medium term. Following standard post-operative treatment with topical antibiotics and topical steroids, the patient had a quiet eye with a functional improvement in vision from hand movements to $6 / 9$.

Concurrent to the patient's ocular oncology referral, a new neck mass was found and lead to further investigation by his medical oncologist. Cerebral frontal lobe and pharyngeal metastases were detected. In light of the confirmed widespread distant metastases, the patient was commenced on palliative targeted immunotherapy in an effort to preserve quality of life, if not prolong it. Immunotherapy was commenced after the vitrectomy and so did not contribute to the ophthalmic presentation.

Given the patient's poor prognosis, it was decided not to undertake external beam radiotherapy to the eye to treat residual intraocular neoplastic disease. He remains under ophthalmic surveillance locally, but to date, he has not needed further treatment for the ICMM.

\section{Discussion}

This case is notable for several reasons. The patient had a functional, comfortable eye, with metastasis from a distant cutaneous melanoma detected most clearly on the posterior lens capsule - a constellation of findings that has never before been described. The clinical diagnosis was confirmed for the first time without enucleation, by 
Fig. 1. Clinical imaging. a Slit lamp photograph of the right eye using retroillumination through the pharmacologically dilated pupil showing brown posterior capsular opacity within the visual axis. b Slit lamp photograph of the unaffected left eye using retroillumination through the pharmacologically dilated pupil showing quiet pseudophakia with minimal capsular opacification. c Slit lamp photograph of the right eye showing brown pigmented cutaneous malignant melanoma metastatic deposits on the anterior (thin arrow) and posterior (thick arrow) lens capsule. d Colour photograph of the inferior iridocorneal angle of the right eye showing pigmented cells deposited in the trabecular meshwork (arrow). e Ultrasound biomicroscopy of the anterior segment showing deposits on the zonules and lens capsule surrounding the intraocular lens implant. The hyperreflective area on the corneal endothelial area is artefact. $\mathbf{f} \mathrm{B}$ scan ultrasonography in T3 position showing complete posterior vitreous detachment, cellular vitreous, and an absence of discrete intraocular mass.
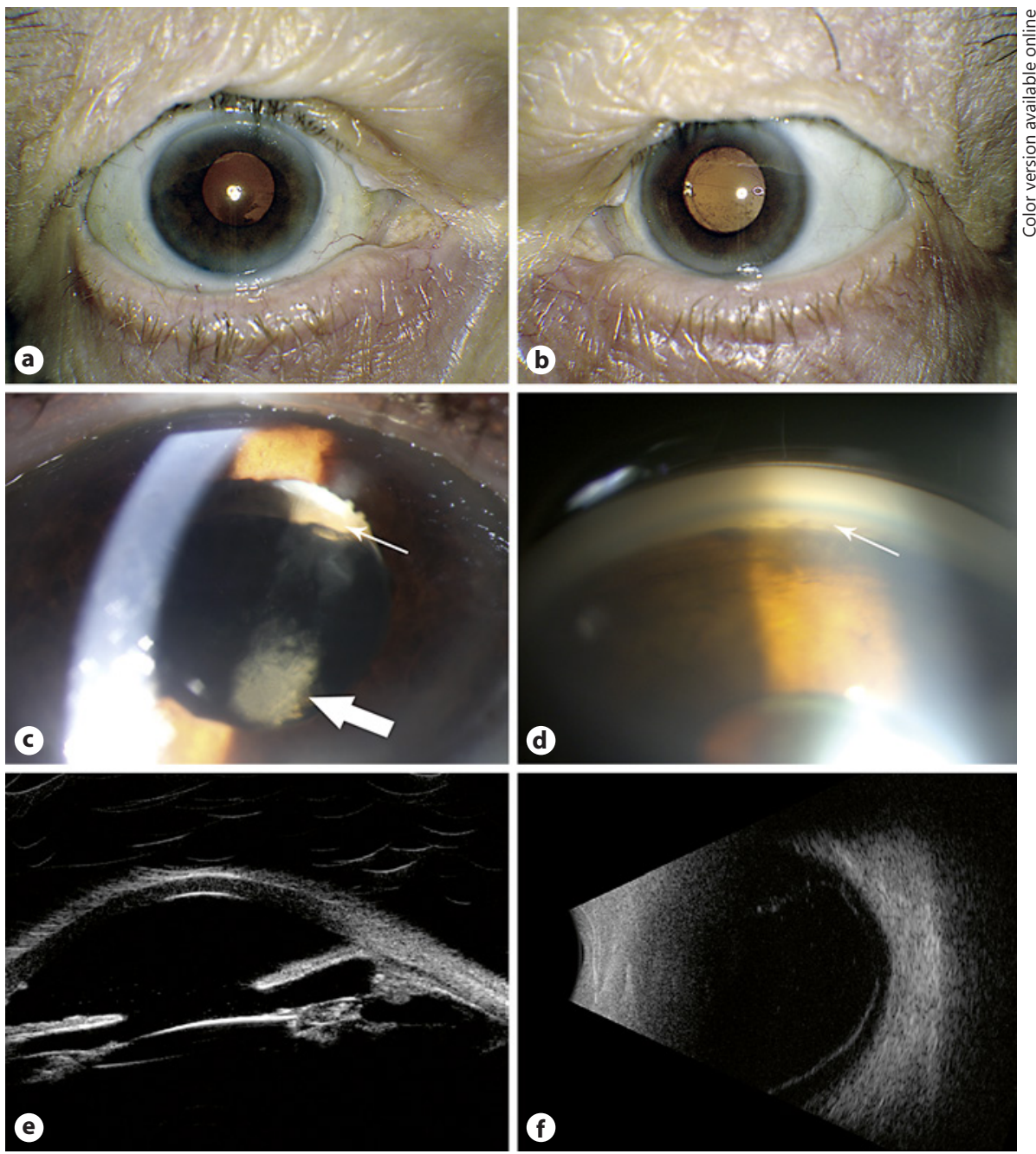

full diagnostic vitrectomy and surgical posterior capsulotomy, despite prior non-diagnostic core vitreous biopsy. This supports previous work indicating the superiority of PPV over core vitreous sampling, particularly in cases suspected of being masquerade uveitis syndromes [5].

The mechanism by which cutaneous melanoma metastasizes to intraocular structures is undoubtedly haematological, yet the lens capsule is avascular. The authors believe that this case represents a diffuse ICMM initially to the intraocular humours, which are relatively common sites of intraocular metastasis [1], and would be similar to those cases already described [2-4]. Free-floating malignant cells within the humours could then have become adherent to the capsule and proliferated, giving rise to brown capsular opacification. This hypothesis is supported by the initial presentation in this case mimicking uve-

Cutaneous Melanoma Metastasis to the Lens Capsule itis, and the detection of pigmented cells in the iridocorneal angle.

The histopathological findings in this case were also unusual; definite granulomas were identified within the vitreous sample. This provides satisfying clinico-histopathological correlation as the patient presented with granulomatous keratic precipitates and intraocular inflammation. There are several potential causes for this inflammatory backdrop which will be discussed.

It is well known that melanoma is highly immunogenic [6]; therefore, it is likely that the intraocular malignant melanoma cells provoked a reactive inflammatory response to their presence. That being said, granulomas are not frequently seen in this context, though granulomas have been described rarely in the stroma of malignant melanoma tumours, the mechanism of which is not fully understood [7]. 
Fig. 2. Histopathology slides from the vitrectomy and capsulotomy specimen. a Haematoxylin and eosin (H\&E) stained cell block cytology preparation showing pieces of lens capsule (arrows) in amongst pigmented and non-pigmented cells. b Higher power $\mathrm{H} \& \mathrm{E}$ of plate a showing some cytologically atypical cells. Note the binucleate atypical cell towards the top left of the plate. c Melan A immunohistochemistry showing that some of the pigmented and non-pigmented cells are Melan A positive (red = positive). d Sox10 immunohistochemistry showing nuclear positivity of some of the cells (small arrows). The larger arrow points to a structure that is suspicious for a granuloma. e CD68 immunohistochemistry showing central confluent staining of several cells in a cluster, confirming the presence of granulomatous inflammation.

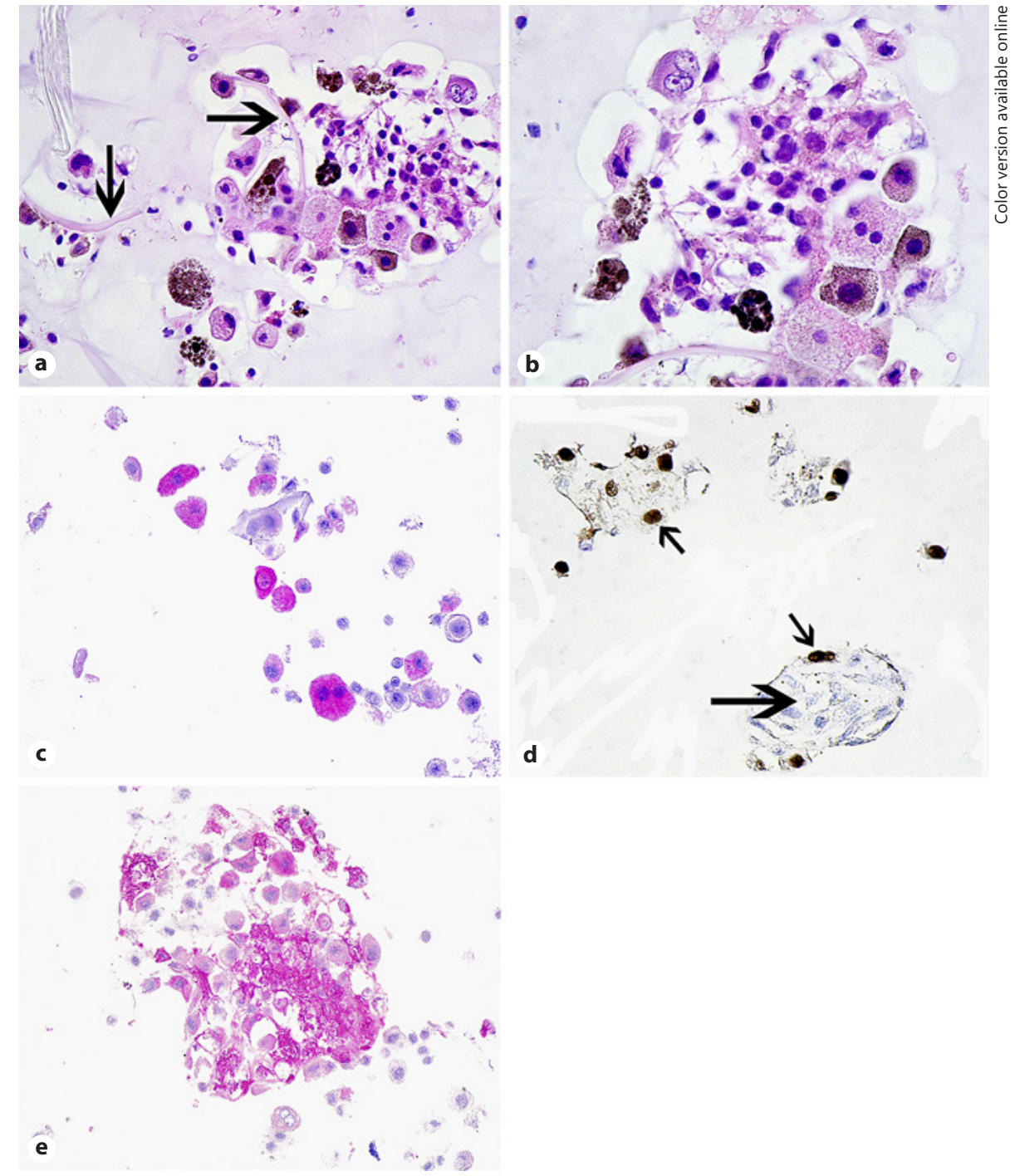

Paraneoplastic granulomatous intraocular inflammation has been described, but not in association with cutaneous melanoma. As with other paraneoplastic phenomena, the mechanism involves an altered immune response activated by neoplasia remote to the eye. The cases in the literature (15 in total) have been described in association with haematological malignancy or systemic carcinoma $[8,9]$. Cytological analysis in those cases established the presence of inflammatory cells and granulomas but did not necessarily reveal malignant cells within the eye, although micro-metastases were detected in some cases. The mechanism was postulated to be secondary to an induced T-cell-mediated host response to circulating tumour antigens [10]. Although the most likely mechanism in our case was di- rect immunogenesis causing granulomatous inflammation, it is possible that the patient had paraneoplastic granulomatous uveitis alongside or preceding detectable intraocular metastasis.

Other potential causes of intraocular granulomatous inflammation exist, but are somewhat less likely given the clinical history. Phacolysis can induce a granulomatous response; however, the patient had quiet pseudophakia for many years preceding presentation, rendering this very unlikely. Immunotherapeutic agents used in the treatment of malignant melanoma are increasingly associated with intraocular inflammation [11]; however, vitreous biopsy in our case preceded any use of immunotherapy by the patient. Finally, the patient could have an associated granulomatous pathology, such as sarcoidosis, 
which has been described in the literature in melanoma patients $[12,13]$. However, our case had no other signs or symptoms to suggest systemic sarcoidosis, rendering this possibility less likely.

This case contributes to the body of literature, expanding what is known regarding the presentation of ICMM and intraocular granulomatous inflammation. The underlying mechanisms are not known with certainty, and further study is needed. As ophthalmologists, we must be aware of where our findings can guide systemic treatment but also be pragmatic in our choice of ocular therapy. Given the poor prognosis in this case, vitrectomy and capsulotomy were both diagnostic and therapeutic, restoring excellent visual acuity almost immediately, and hopefully for the rest of the patient's life.

\section{Acknowledgements}

The authors wish to thank Josie Stephenson and Lisa Macdonald for their help with accessing medical records. We would also like to thank Kim Foster and Thomas Evans for their assistance with clinical imaging.

\section{Statement of Ethics}

This research was conducted ethically in accordance with the World Medical Association Declaration of Helsinki. The patient gave written informed consent for publication of the case. Ethical approval was not required for this purely observational retrospective case report.

\section{Disclosure Statement}

The authors have no conflicts of interest to declare.

\section{Funding Sources}

The authors have no funding to declare.

\section{Author Contributions}

H.Q. wrote the manuscript and collected data on the case. H.S.M. wrote the histopathology section of the manuscript, contributed to the discussion, and edited the manuscript. K.S.C. provided vitreo-retinal consult and edited the manuscript. I.G.R. produced the idea for the paper and edited the manuscript.

\section{References}

1 Rosenberg C, Finger PT. Cutaneous malignant melanoma metastatic to the eye, lids, and orbit. Surv Ophthalmol. 2008 May-Jun;53(3): 187-202.

2 Bowman CB, Guber D, Brown CH 3rd, Curtin VT. Cutaneous malignant melanoma with diffuse intraocular metastases. Arch Ophthalmol. 1994 Sep;112(9):1213-6.

3 Gündüz K, Shields JA, Shields CL, Eagle RC Jr. Cutaneous melanoma metastatic to the vitreous cavity. Ophthalmology. 1998 Apr; 105(4):600-5.

4 Solomon JD, Shields CL, Shields JA, Eagle RC Jr. Posterior capsule opacity as initial manifestation of metastatic cutaneous melanoma. Graefes Arch Clin Exp Ophthalmol. 2011 Jan; 249(1):127-31.
5 Patel DS, Khan IJ, Zayed MG, Partridge D, Rennie IG, Mudhar HS, et al. Full diagnostic vitrectomy with posterior vitreous detachment for the diagnosis of vitritis due to uncertain etiology. Retina. 2019 Oct;39(10):18729.

6 Passarelli A, Mannavola F, Stucci LS, Tucci M, Silvestris F. Immune system and melanoma biology: a balance between immunosurveillance and immune escape. Oncotarget. 2017; 8(62):106132-42. https://doi.org/10.18632/ oncotarget.22190.

7 Banerjee SS, Harris M. Morphological and immunophenotypic variations in malignant melanoma. Histopathology. 2000 May;36(5): 387-402.

8 McGrath LA, Mudhar HS, Sheard R, SpiteriCornish K, Winder S, Rundle P, et al. Paraneoplastic Granulomatous Vitritis: elaboration of 8 Cases. Ophthalmol Retina. 2019 Jul; 3(7):589-96.
9 Balasubramaniam SC, Salomao DR, Davies JB, et al. Paraneoplastic sarcoid-like reactions and the eye. Retina 2015 Apr;35(4):789-97.

10 Brincker H. Sarcoid reactions in malignant tumours. Cancer Treat Rev 1986 Sep;13(3): 147-56.

11 Dimitriou F, Frauchiger AL, Urosevic-Maiwald M, Naegeli MC, Goldinger SM, Barysch M, Franzen D, Kamarachev J, Braun R, Dummer R, Mangana J. Sarcoid-like reactions in patients receiving modern melanoma treatment. Melanoma Res. 2018 Jun;28(3):230-6.

12 Beutler BD, Cohen PR. Sarcoidosis in melanoma patients: case report and literature review. Cancers (Basel) 2015 Jun;7(2):1005-21.

13 Seve P, Schott AM, Pavic M, Broussolle C, Gilis L, Thomas L. Sarcoidosis and melanoma: a referral center study of 1,199 cases. Dermatology 2009;219(1):25-31. 